\title{
Evaluación de la posición condilar y de las vías aéreas en niños con mordida cruzada posterior unilateral antes y después de tratamiento con férula de expansión. Parte 1
}

Evaluation of the condylar and airway position in children with unilateral posterior crossbite before and after treatment with an expansion splint. Part 1

Avaliação da posição condilar e das vias aéreas em crianças com mordida cruzada posterior unilateral antes e após tratamento com tala expansora. Parte 1

\section{Jesús Alberto Hernández-Silva ${ }^{1}$ Carolina Rodríguez-Manjarrés ${ }^{2}$}

Recibido: 21 de octubre de 2020

Aprobado: 28 de agosto de 2021 Publicado: 22 de octubre de 2021

Cómo citar este artículo: Hernández-Silva JA, Rodríguez-Manjarrés C. Evaluación de la posición condilar y de las vías aéreas en niños con mordida cruzada posterior unilateral antes y después de tratamiento con ferula de expansión. Parte 1. Revista Nacional de Odontología. (2021); 17(1), 1-23. doi: https://doi.org/10.16925/2357-4607.2021.01.07

Artículo de investigación. https://doi.org/10.16925/2357-4607.2021.01.07

1 Escuela de Odontología, Universidad del Valle, Cali, Colombia.

Email: jesus.hernandez@correounivalle.edu.co

ORCID: https://orcid.org/0000-0003-3984-6639

2 Escuela de Odontología, Universidad del Valle, Cali, Colombia.

ORCID: https://orcid.org/0000-0003-4328-0156 
2 Evaluación de la posición condilar y de las vías aéreas en niños con mordida cruzada posterior unilateral antes y después de tratamiento con férula de expansión. Parte 1

\title{
Resumen
}

Introducción: la etiología de la mordida cruzada posterior (MCP), incluye componentes dentales, esqueléticos, musculares y funcionales; puede ser unilateral o bilateral. Se reconoce ampliamente la importancia del tratamiento oportuno y la expansión rápida maxilar (ERM) es una alternativa para ampliar el arco dental superior, genera una mejoría en las condiciones de ventilación de los niños mejorando su calidad de vida y previniendo condiciones médicas futuras.

Objetivo: observar en tomografías computarizadas ConeBeam (TC-CB) cambios en posición condilar y dimensiones de vías aéreas, antes, durante y después de 6 meses de ERM para corregir la mordida cruzada posterior unilateral (MCPU).

Metodología: nueve pacientes, de 6 a 10 años, dentición mixta temprana, con MCPU,sin anomalías dentales fueron tratados con ERM por 3 meses y 3 más de retención; tomografías bimaxilares pre y postratamiento fueron tomadas para evaluar cambios de posición condilar y dimensionales de vías aéreas.

Resultados: el lado cruzado presentó aumento del espacio anterior de cóndilo, disminución leve de la longitud superior y una marcada disminución de la longitud posterior entre cóndilo y fosa glenoidea, evidenciando un desplazamiento posterior, confirmando una posición posterior, después de realizada la ERM. El lado no cruzado presentó el mismo comportamiento. A nivel del volumen de las vías aéreas no se presentaron cambios estadísticamente significativos.

Conclusiones: la ERM es efectiva para el tratamiento de la MCP en dentición mixta ya que cambia la forma del maxilar, genera cambios en posición condilar con resultados estables en el tiempo que favorecen el desarrollo armónico del complejo craneofacial.

Palabras clave: expansión rápida maxilar; mordida cruzada posterior unilateral; posición condilar; dimensión vías aéreas; TC-CB.

\begin{abstract}
Introduction: the etiology of posterior crossbite (PCM) includes dental, skeletal, muscular and functional components; It may be unilateral or bilateral. The importance of timely treatment is widely recognized and rapid maxillary expansion (ERM) is an alternative to expand the upper dental arch, it generates an improvement in the ventilation conditions of children, improving their quality of life and preventing future medical conditions.

Objective: to observe in ConeBeam computed tomography (CB-CT) changes in condylar position and airway dimensions, before, during and after 6 months of MRE to correct the unilateral posterior crossbite (MCPU).

Methodology: nine patients, 6 to 10 years old, with early mixed dentition, with MCPU, without dental anomalies, were treated with ERM for 3 months and 3 more months of retention; Pre- and post-treatment bimaxillary tomographies were taken to evaluate condylar position and dimensional changes of the airways.

Results: the crossed side presented an increase in the anterior condyle space, a slight decrease in the superior length and a marked decrease in the posterior length between the condyle and the glenoid fossa, showing a posterior displacement, confirming a posterior position, after the MRA was performed. The non-crossed side presented the same behavior. At the level of airway volume, there were no statistically significant changes.

Conclusions: ERM is effective for the treatment of CPM in mixed dentition since it changes the shape of the maxilla, generates changes in condylar position with stable results over time that favor the harmonious development of the craniofacial complex.
\end{abstract}

Keywords: rapid maxillary expansion; unilateral posterior crossbite; condylar position; airway dimension; $\mathrm{TC}-\mathrm{CB}$ 


\section{Resumo}

Introdução: a etiologia da mordida cruzada posterior (MCP) inclui componentes dentários, esqueléticos, musculares e funcionais; Pode ser unilateral ou bilateral. A importância do tratamento oportuno é amplamente reconhecida e a expansão rápida da maxila (ERM) é uma alternativa para expandir a arcada dentária superior, pois gera uma melhora nas condições de ventilação das crianças, melhorando sua qualidade de vida e prevenindo futuras condições médicas.

Objetivo: observar na tomografia computadorizada ConeBeam (CB-CT) alterações na posição condilar e dimensões das vias aéreas, antes, durante e após 6 meses de ERM para correção da mordida cruzada posterior unilateral (PUMC).

Metodologia: nove pacientes, de 6 a 10 anos, dentição mista precoce, com MCPU, sem anomalias dentárias foram tratados com ERM por 3 meses e mais 3 retenções; Tomografias computadorizadas bimaxilares foram realizadas antes e após o tratamento para avaliar as mudanças na posição condilar e nas dimensões das vias aéreas.

Resultados: o lado cruzado apresentou aumento do espaço anterior do côndilo, discreta diminuição do comprimento superior e acentuada diminuição do comprimento posterior entre o côndilo e a fossa glenoidal, evidenciando um deslocamento posterior, confirmando uma posição posterior, após realização do MRE. O lado não cruzado apresentou o mesmo comportamento. Ao nível do volume das vias aéreas, não houve alterações estatisticamente significativas.

Conclusões: A MRE é eficaz para o tratamento da MCP na dentição mista, pois altera a forma da maxila, gera mudanças na posição condilar com resultados estáveis ao longo do tempo que favorecem o desenvolvimento harmonioso do complexo craniofacial.

Palavras-chave: expansão rápida da maxila; mordida cruzada posterior unilateral; posição condilar; dimensão das vias aéreas; CT-CB.

\section{Introducción}

En Colombia, las maloclusiones tienen una prevalencia del $46,9 \%$ en los niños a los 5 años, y rangos de $56,1 \%$ y 60,8 \% a los 12 y 15 años respectivamente, según el último Estudio Nacional de Salud Bucal - ENSAB IV, realizado en el $2014(1,2)$.

La mordida cruzada posterior (MCP) es un término que describe una anomalía de la oclusión en sentido transversal. Presenta una relación vestibulo-lingual inversa entre dientes antagonistas durante la oclusión, que involucra al menos un diente posterior, molar o premolar (3-5). Puede presentarse de manera unilateral o bilateral, y los reportes de su prevalencia varían entre el $2,7 \%$ y $23 \%$ (6). Se acepta que en su etiología hay involucrados factores genéticos como hipoplasia del maxilar, hiperplasia mandibular y alteraciones cráneo-faciales relacionadas con síndromes. Factores ambientales como hábitos de succión no nutritiva o uso de chupo, deglución atípica que genera una posición inadecuada de la lengua. Respiración nasal alterada por obstrucción en las vías aéreas o alergias, también hipertrofia de amígdalas y adenoides y en general factores que afectan el diámetro transversal del arco dental superior $(4,7,8,9)$. 
4 Evaluación de la posición condilar y de las vías aéreas en niños con mordida cruzada posterior unilateral antes y después de tratamiento con férula de expansión. Parte 1

También existen factores funcionales tales como el desplazamiento de la mandíbula, debido a interferencias oclusales que se encuentran altamente asociadas a los caninos en la dentición primaria, a la erupción del primer molar en la dentición mixta temprana y con la retención prolongada de algún diente primario en la dentición mixta tardía, estas situaciones provocan cambios e inestabilidad en la oclusión (6,7, 9) (figuras 1 A-E).
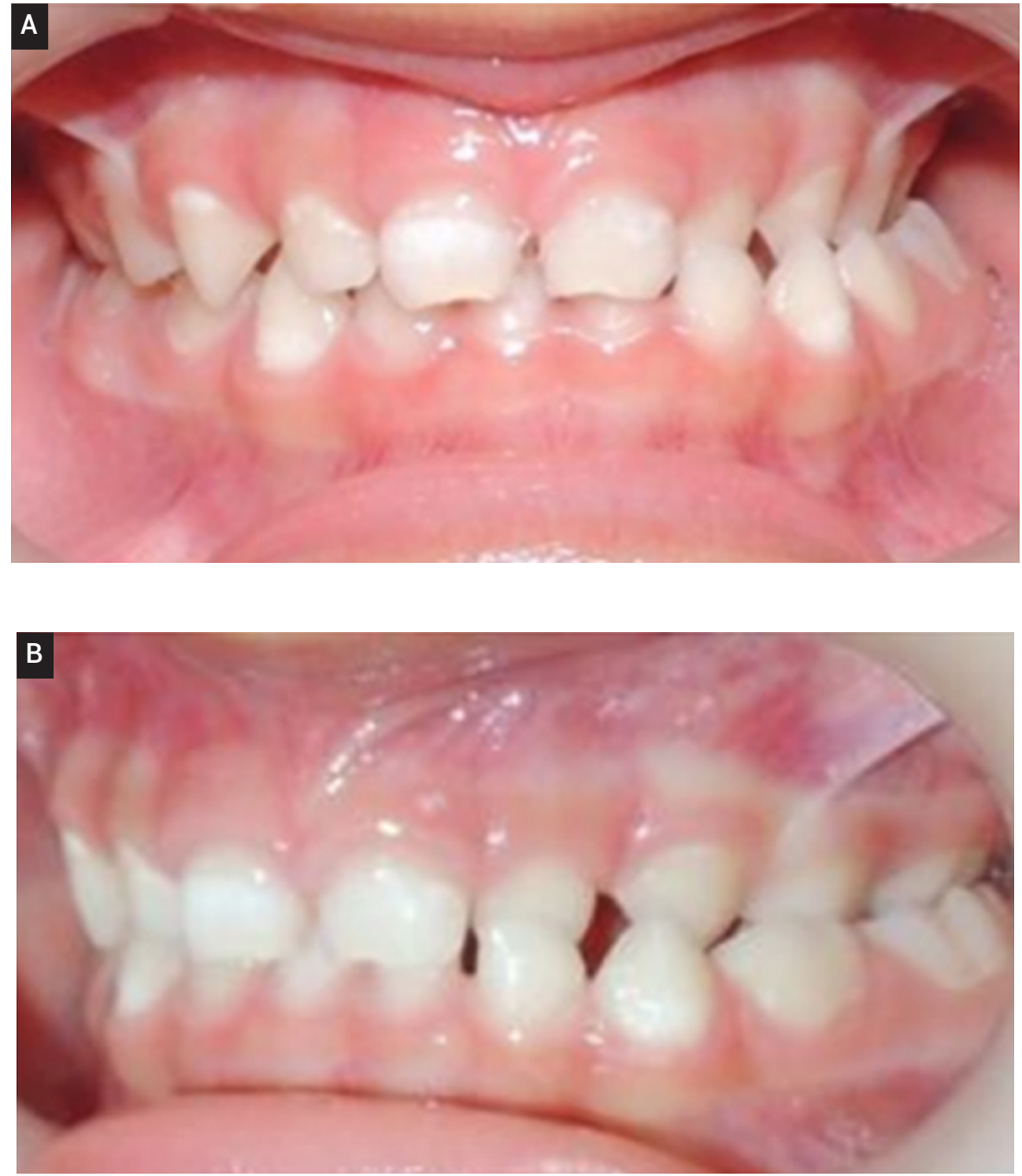

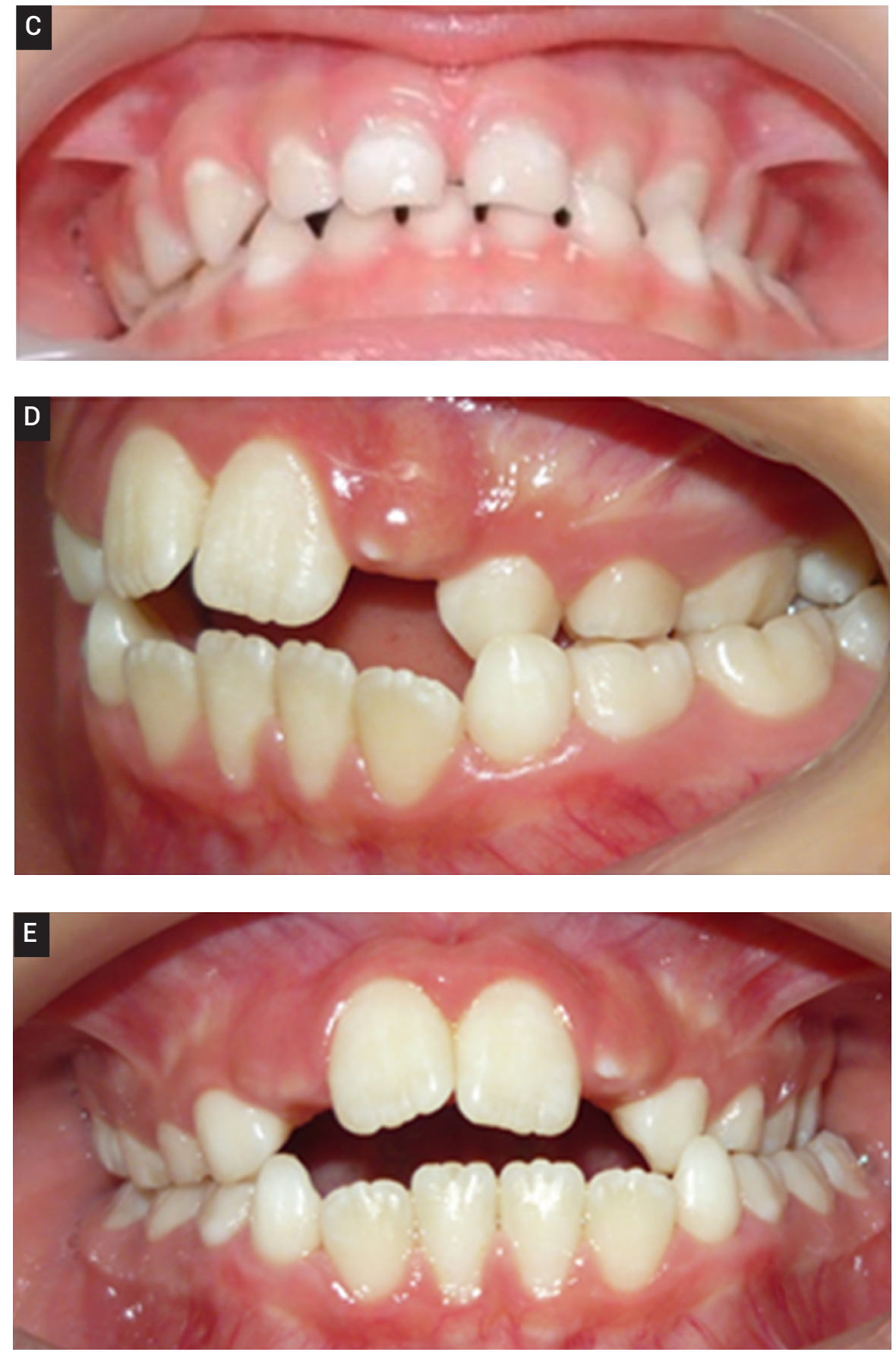

Figura 1. A. B. C. Fotografías intraorales dentición primaria, donde se evidencia una mordida cruzada posterior unilateral izquierda y desviación de la línea media dental inferior. Figuras 1. D.E. Fotografías intraorales dentición mixta, donde se evidencia una mordida cruzada posterior unilateral izquierda, mordida abierta anterior, desviación de la línea media dental inferior e interferencias oclusales altamente asociadas a los caninos primarios.

Fuente: Elaboración propia. 
6 Evaluación de la posición condilar y de las vías aéreas en niños con mordida cruzada posterior unilateral antes y después de tratamiento con férula de expansión. Parte 1

Es reconocido que las alteraciones durante el desarrollo de la dentición primaria y mixta afectarán posteriormente el estado de la oclusión permanente y, en el caso de la mordida cruzada posterior, se ha descrito una posición asimétrica de los cóndilos mandibulares en posición dental de máxima intercuspidación. Por tanto, se considera que es importante tratar esta maloclusión una vez identificada ya que la corrección espontánea es inusual $(6,9,10)$.

El tratamiento oportuno evitará alteraciones en las diferentes estructuras del sistema estomatognático. De no ser tratada se puede presentar con el tiempo un desarrollo óseo asimétrico de la rama mandibular del lado afectado, ocasionando asimetrías mandibulares o faciales, desórdenes a nivel de la articulación temporomandibular (ATM), cambios morfológicos y de posición en el cóndilo mandibular, encontrándose el cóndilo del lado afectado en una posición más posterior y superior con respecto al lado contralateral $(4,6,10,11)$.

La estabilidad y funcionalidad de la oclusión puede afectarse, ya que se altera la actividad de los músculos, observándose una reducción del tono muscular y de la fuerza masticatoria, que lleva a una disminución del espesor del músculo del lado afectado, patrón de preferencia masticatorio por el lado cruzado y una tendencia a ciclos irregulares ocasionados por el desplazamiento mandibular y desviación de la línea media dental (10-12).

Para esta maloclusión que afecta el desarrollo del plano transversal, limitando el ancho del arco superior, la expansión maxilar es una alternativa terapéutica aceptada (13). Esta expansión puede realizarse de manera rápida (ERM) o de manera lenta o controlada y puede realizarse con aparatología fija o removible. Diversa aparatología se ha propuesto para aumentar el ancho del arco superior, por ejemplo, tornillos tipo Hyrax, Hass, Quad-Helix, SuperScrew, Pistas Directas e Indirectas Planas (PDP - PIP), placas de Hawley modificadas, entre otros $(6,7,14-17)$.

El uso de un tornillo tipo Hyrax embebido en una férula de acrílico (figura 2), es una de las opciones de aparatología fija para realizar una expansión rápida maxilar (ERM), esta férula cuenta con una altura de $3 \mathrm{~mm}$ aproximadamente, y se extiende desde el canino o primer molar primario hasta el último molar en boca completamente erupcionado. Se realiza una activación del tornillo 1/4 de vuelta al día, 4 veces por semana, hasta lograr la corrección de la MCP unilateral durante aproximadamente 4 a 6 semanas $(4,7,8,18)$. 

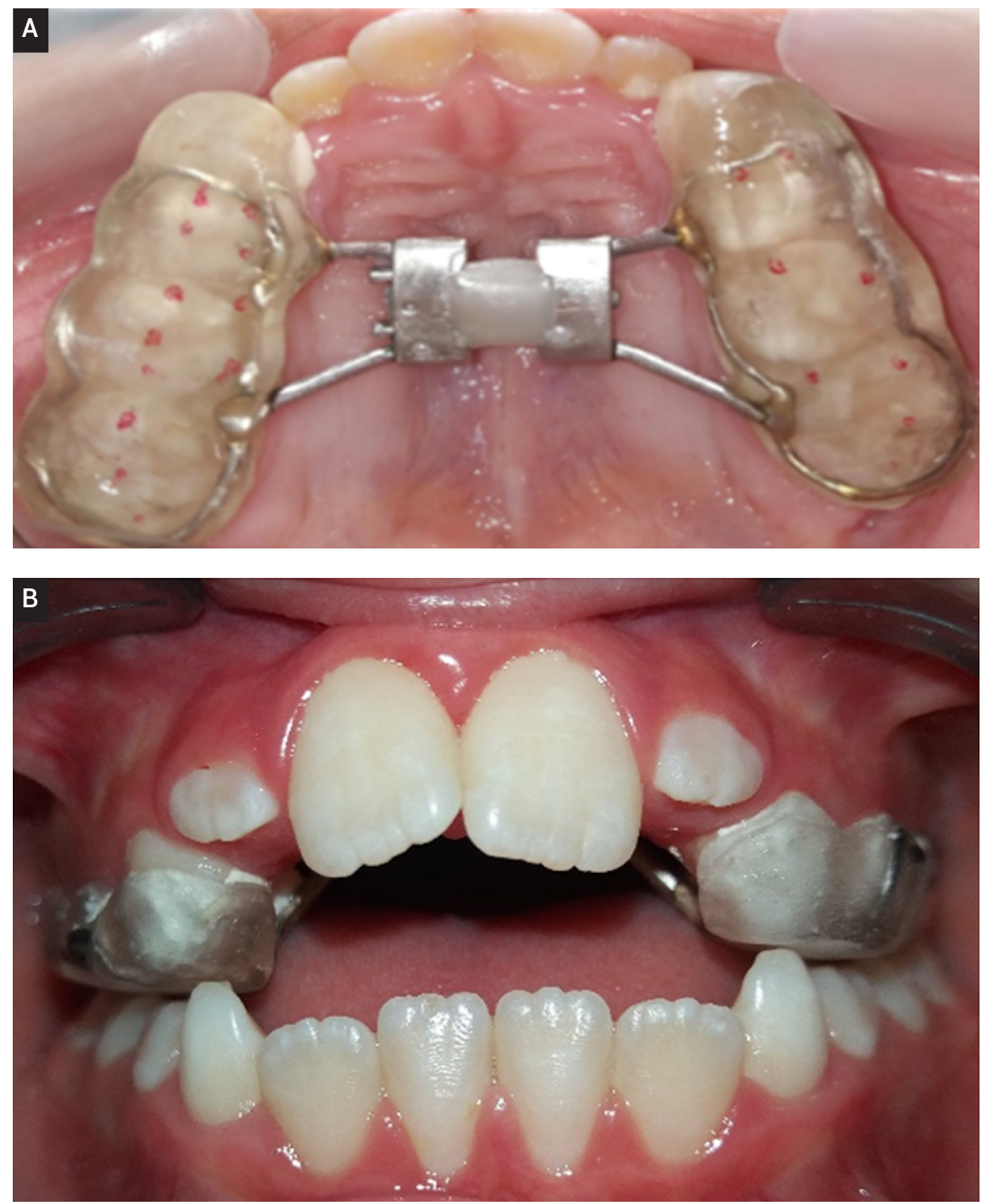

Figura 2. A.B. Fotografías intraorales que permiten observar férula de expansión en acrílico como alternativa terapéutica para la mordida cruzada posterior unilateral o bilateral. Fuente: elaboración propia.

La ERM es utilizada para aumentar el ancho del arco superior ya que genera una disyunción de la sutura media palatina y cambios dentoalveolares en la inclinación de molares y premolares. Adicionalmente, y de manera indirecta, ocurren cambios como un aumento moderado en el espacio de la vía aérea superior desde la espina nasal posterior hasta el punto basion. También se dan cambios mandibulares asociados a 
8 Evaluación de la posición condilar y de las vías aéreas en niños con mordida cruzada posterior unilateral antes y después de tratamiento con férula de expansión. Parte 1

la ERM, pues ella se desplaza acompañando los cambios del maxilar superior y los cóndilos se acomodan en las fosas glenoideas mejorando la asimetría que presentaba antes de iniciado el tratamiento $(4,6,10,14,18-21)$.

Aunque el auge de los estudios tridimensionales empleando tomografías computarizadas tipo Cone Beam (TC-CB) va en aumento, pocos estudios se han realizado para evaluar cambios condilares y de la vía aérea superior después de realizar un tratamiento de expansión rápida maxilar para la corrección de mordidas cruzadas posteriores unilaterales en dentición primaria o mixta temprana $(10,12,22)$.

El propósito de este estudio es observar en tomografías computarizadas tipo Cone Beam (TC-CB) los cambios que puedan ocurrir en la posición condilar y a nivel de la ATM. Así como el comportamiento de las dimensiones de las vías aéreas en pacientes con un rango de edad de 6 a 10 años antes, durante y después de 6 meses de tratamiento de expansión rápida maxilar como alternativa terapéutica para la mordida cruzada posterior unilateral.

\section{Materiales y métodos}

Se realizó un estudio de serie de casos, en el cual se seleccionaron nueve pacientes niños con un rango de edad de 6 a 10 años que presentaran mordida cruzada posterior unilateral en dentición mixta, sanos, sin anomalías dentales. Se excluyeron los pacientes con alteraciones craneofaciales y condiciones médicas que afectan el sistema estomatognático.

Los pacientes pertenecían a la clínica de Ortopedia Maxilar del programa de posgrado de Odontología Pediátrica y Ortopedia Maxilar de la Universidad del Valle (Cali-Colombia), se notificó a los padres acerca del tratamiento, así como sus ventajas y desventajas. Se firmó previamente el consentimiento informado por los padres donde aceptaban la participación de los menores en la investigación. Ninguno de ellos había recibido tratamiento previo de ortopedia maxilar. Este estudio cuenta con el aval del Comité Institucional de Revisión de Ética Humana y Experimentación Animal de la Facultad de Salud de la Universidad del Valle (Cali -Colombia). Se cumplió igualmente con lo dispuesto en la Declaración de Helsinki y la Resolución No. 008430 de 1993 del Ministerio de Salud para investigación con seres humanos.

Todos los pacientes fueron tratados con expansión rápida maxilar con aparatología tipo Hyrax tornillo de $9 \mathrm{~mm}$ embebido en férula de acrílico de $3 \mathrm{~mm}$ de altura en promedio, con extensión desde el canino superior primario hasta el primer molar permanente superior. Previo a la cementación, se realizó profilaxis y posteriormente se aplicó vaselina en todas las superficies dentales para crear un aislamiento. La 
férula fue cementada con Ionómero cementante fuji tipo I marca GC®. Se retiraron excesos de material, se verificó oclusión y retiro de posibles interferencias con papel de articular (figura 2.A). Se dieron instrucciones sobre uso y medidas de higiene.

El primer control se realizó ocho días después de la cementación, y posteriormente se evaluaron cada tres semanas. El protocolo de activación para el tornillo fue de $1 / 4$ de vuelta cada dos días durante tres meses (0,25 mm por 1/4). El tiempo de tratamiento activo fue de tres meses en promedio, la maloclusión se corrigió satisfactoriamente en todos los casos y se mantuvo la aparatología por tres meses como contención.

Para observar los cambios que pueden ocurrir en la posición condilar de la ATM se tomaron tomografías bimaxilares computarizadas con técnica Cone Beam. También se utilizaron las tomografías para analizar el comportamiento en el volumen de las vías aéreas superiores, después de realizada la expansión rápida maxilar, en niños con mordida cruzada posterior unilateral. Estas imágenes fueron realizadas en el equipo I-CAT 17-19®, en dos momentos del estudio: al inicio ( $\mathrm{T} 1$ ) y 6 meses después del tratamiento (T2), con retiro previo de la férula.

Todas las imágenes tomográficas fueron tomadas por el mismo operador y el proceso se estandarizó para mayor confiabilidad en medidas y resultados de la siguiente manera: paciente sentado, la cabeza se estabilizó para obtener el plano de Frankfurt paralelo al piso y permanecer en máxima intercuspidación durante la toma. Continuando con la estandarización, una vez obtenida la imagen, una especialista experimentada en radiología valoró y midió las variables de interés, y se calcularon coeficientes de correlación intraclase (ICC) para las medidas dimensionales (espacio anterior, superior, posterior y posición expresado en $\mathrm{mm}$ ) y el coeficiente kappa para la variable categórica de la posición del punto p (correspondiente a la concentricidad absoluta del cóndilo expresada en porcentaje). Se aceptó un ICC estadísticamente significativo y kappa superior a 0,8 intra e inter observador. Para continuar este proceso y minimizar posibles errores de medición debido a la ausencia de un cefalostato, las imágenes en 3D se reorientan de acuerdo con dos planos de referencia.

\section{Estandarización de la posición tomográfica:}

Se tomó como referencia el estudio publicado por Leonardi et al. (23), en el cual se reorientan las imágenes 3D de acuerdo con 2 planos de referencia: primero, el plano NFZ, un plano frontal que pasa a través de las dos suturas frontocigomáticas en el borde interno de la órbita y nasion; y segundo, el plano de Frankfurt (FH). Ambos 
Evaluación de la posición condilar y de las vías aéreas en niños con mordida cruzada posterior unilateral antes y después de tratamiento con férula de expansión. Parte 1

planos deben ser paralelos al borde superior e inferior de la pantalla, esto mejora la fiabilidad y consistencia de los resultados al momento de realizar las medidas condilares (23).

\section{Medidas tomográficas:}

Se tomó como referencia estudio de Ikeda y Kawamura (22). Partiendo de una posición estándar se realizó:

Localización de los cóndilos en el plano axial y frontal, teniendo en cuenta la zona media de ambos cóndilos para tomar las medidas.

En el plano sagital, para medir la posición condilar, se utilizó la línea horizontal verdadera (THL por sus siglas en inglés) como plano de referencia paralelo a FH ubicándolo en el punto más profundo de la cavidad glenoidea.

Partiendo del punto más profundo de la cavidad glenoidea, se trazan líneas tangentes a los aspectos anterior y posterior más prominentes del cóndilo.

Se tomaron 3 medidas de los espacios articulares en milímetros: el espacio articular superior se midió desde el aspecto más superior del cóndilo a la parte más profunda de la fosa glenoidea; las distancias desde los puntos tangentes anterior y posterior a la fosa glenoidea se midieron como el espacio articular anterior; y el espacio articular posterior (figura 3.A-B).

Se verifica la medida del espacio articular superior, haciendo la medición en el plano coronal (22).

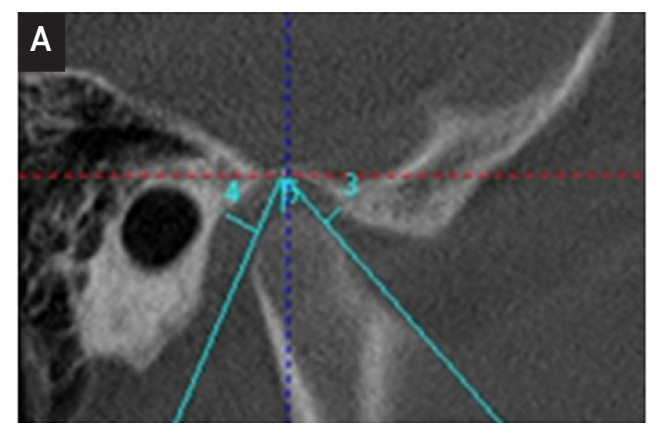

Figura 3. A. Esquema que representa los puntos de referencia y medidas lineales del espacio entre el cóndilo y la fosa glenoidea. Se observa como la línea horizontal verdadera (THL) se utiliza como plano de referencia. La distancia desde el punto más superior del cóndilo (SC) hasta el aspecto más superior de la fosa glenoidea (SF) en la THL se mide como el espacio articular superior (SS). Líneas tangentes a los aspectos anteriores y posteriores más prominentes del cóndilo son trazados desde la fosa glenoidea (SF). Las distancias desde los puntos tangentes anterior (AC) y posterior (PC) a la fosa glenoidea se miden como el espacio anterior (AS) y posterior (PS) de la articulación respectivamente.

Fuente: (19). 


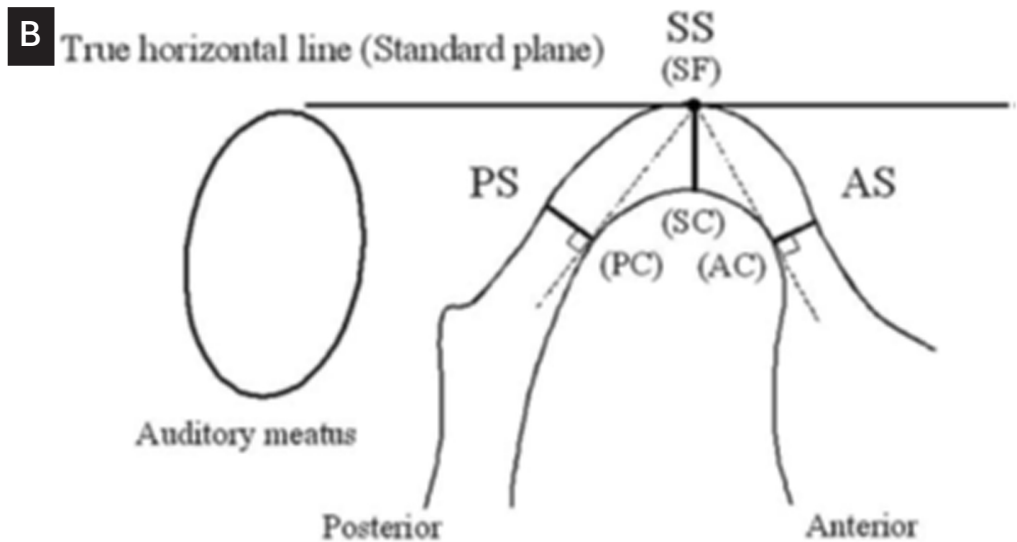

3. B. Imagen tomografica correspondiente a un paciente del estudio, en donde se observan los puntos de referencia y medidas lineales del espacio entre el cóndilo y la fosa glenoidea, previo a realizar medición.

Fuente: elaboración propia.

Para identificar la posición del cóndilo, se usó la formula $\frac{P-A}{P+A} * 100$. Esta fórmula representa el desplazamiento desde la concentricidad absoluta en porcentaje. Una puntuación de 0 indica posición céntrica del cóndilo dentro de la fosa, un valor negativo una posición posterior, y un valor positivo indica una posición anterior del cóndilo en la fosa glenoidea $(24,25)$.

\section{Vías aéreas:}

Para la evaluación de las vías aéreas superiores se hizo una renderización volumétrica delimitando la zona superior con el plano palatino que va desde la espina nasal anterior a la espina nasal posterior, pero en este caso la línea se extendió hasta la pared posterior de la faringe. La zona inferior se delimitó con una línea paralela al plano palatino que pasaba por el punto más superior de la epiglotis. Usando el software TX-estudio se obtuvo el volumen total de la vía aérea (26) (figura 4). 


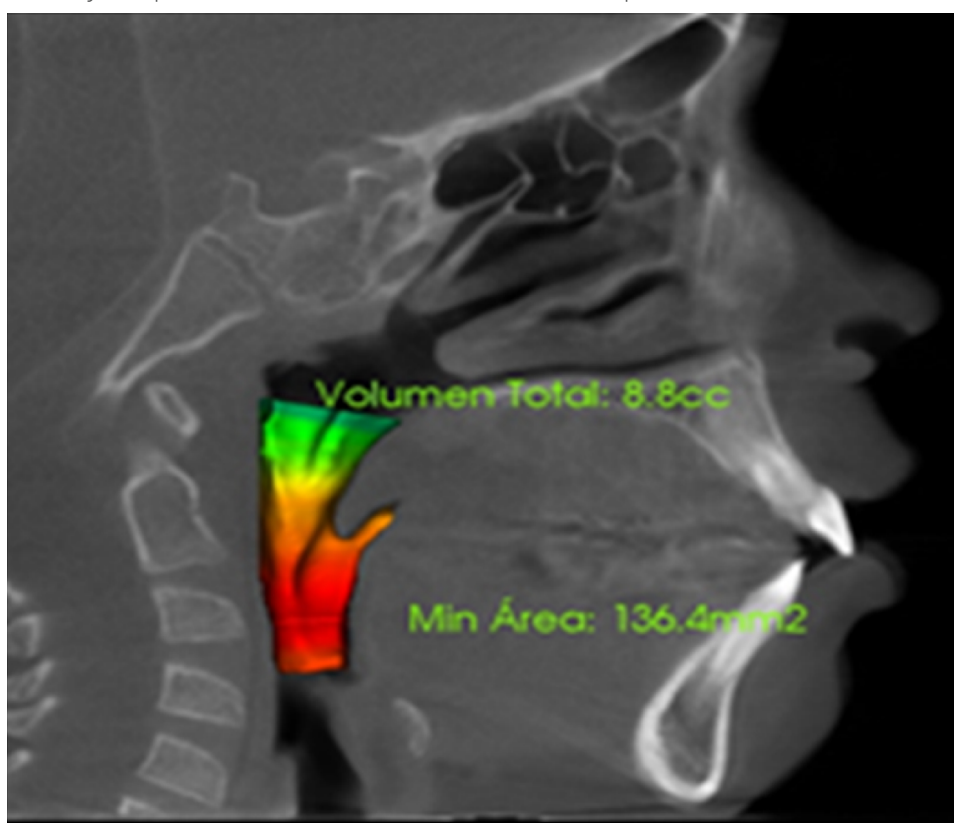

Figura 4. Segmentos en los que se puede dividir la vía aérea superior (nasofaringe, velofaringe e hipofaringe)

Fuente: elaboración propia.

\section{Análisis estadístico:}

Las variables de interés fueron analizadas utilizando el paquete para análisis estadístico STATA IC 15, que permitió calcular las medidas de tendencia central y de dispersión en las variables de espacio condilar (anterior, superior, posterior) y la presentación de frecuencias absolutas y relativas para las variables en escala categórica (nominal u ordinal). También se contrastaron las medidas dimensionales entre el inicio (T1) y el final (T2) mediante la prueba de t de Student pareada. Las pruebas de contraste de hipótesis para variables numéricas se realizaron después de la verificación de normalidad mediante prueba Shapiro-Wilk e igualdad de varianzas con la prueba de Levene. En caso de no cumplir los supuestos de normalidad, se aplicó la prueba de Wilcoxon. Además, se realizó análisis de concordancia mediante coeficiente Kappa para la clasificación de la posición para los lados derecho e izquierdo, así como, para lado alterado (mordida cruzada) o no. Se estableció un nivel de significancia de 0,05 y de confianza del $95 \%$. 


\section{Resultados}

El total de la muestra fueron 9 pacientes, 6 hombres y 3 mujeres, de 6 a 10 años

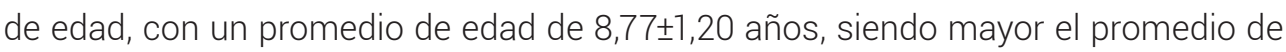
edad de las mujeres ( $9 \pm 1$ años) frente a la edad promedio de los hombres $(8,66 \pm 1,36$ años) sin diferencias estadísticamente significativas ( $p=0,583)$. Todos presentaban un diagnóstico de mordida cruzada posterior unilateral, y recibieron como alternativa terapéutica expansión rápida maxilar (ERM) durante 3 meses y posteriormente 3 meses más de retención o estabilidad de los resultados alcanzados. En el 77,78 \% de los casos (7/9) el lado cruzado fue el derecho y para el sexo femenino la totalidad de casos se diagnosticaron en este mismo lado.

Tabla 1. Frecuencia de mordida cruzada posterior y posición condilar (lado derecho e izquierdo) en $\mathrm{T} 1$ según el sexo.

\begin{tabular}{lccc}
\hline \multirow{2}{*}{$\begin{array}{c}\text { Ubicación Mordida } \\
\text { Cruzada }\end{array}$} & \multicolumn{2}{c}{ Sexo } & Total \\
\cline { 2 - 3 } & Femenino & Masculino & $4(22,22)$ \\
\hline Izquierdo & $0(0,00)$ & $4(33,33)$ & $14(77,78)$ \\
\hline Derecho & $6(100)$ & $8(66,67)$ & \\
\hline Lado Izquierdo T1 & & & $6(66,67)$ \\
\hline Anterior & $1(33,33)$ & $5(83,33)$ & $2(22,22)$ \\
\hline Céntrica & $1(33,33)$ & $1(16,67)$ & $1(11,11)$ \\
\hline Posterior & $1(33,33)$ & $0(0)$ & $6(77,78)$ \\
\hline Lado Derecho T1 & & & $0(0)$ \\
\hline Anterior & $1(33,33)$ & $6(100)$ & $1(22,22)$ \\
\hline Céntrica & $0(0)$ & $0(0)$ & \\
\hline Posterior & $2(66,67)$ & $0(0)$ & \\
\hline
\end{tabular}

Fuente: elaboración propia

Con los datos recolectados, antes del tratamiento de ERM, se encontró que en el lado cruzado existe un aumento del espacio anterior de cóndilo, una disminución leve de la longitud superior y una marcada disminución de la longitud posterior entre el cóndilo y la fosa glenoidea, lo que evidencia un desplazamiento posterior del cóndilo afectado. Este resultado se confirma con los cambios encontrados en los valores de la posición condilar, que tenían al inicio (T1) un valor positivo $(8,435)$ y pasaron a estar negativos en la medida final (T2) (-12,729), exponiendo así una posición posterior del cóndilo después de haber terminado la primera fase del tratamiento. El lado no cruzado presentó el mismo comportamiento del lado del cóndilo afectado (tablas 2 y 3 ). 
Al realizar el análisis del espacio condilar en ambos lados, sin tener en cuenta cuál era el lado cruzado y no, se observó que en el cóndilo izquierdo respecto a la longitud anterior se presentó un aumento entre T1 vs T2 que fue estadísticamente significativo ( $p=0,009)$, así como para el cóndilo del lado derecho, con una significancia $p=0,028$. En la longitud posterior los valores tanto del cóndilo derecho e izquierdo mostraron una disminución, la cual es estadísticamente significativa, con valores de $p=0,003$ y 0,038 respectivamente. Esto permitió confirmar que hubo un desplazamiento hacia el sector posterior de ambos cóndilos (cruzado y no cruzado) en los pacientes, y fue un cambio estadísticamente significativo (valor de $p=0,000$ ) en ambos lados (tabla 2).

Tabla 2. Distancia de espacios condilares derecho (Der) e izquierdo (Izq) en milímetros ( $\mathrm{mm}$ ) antes (T1) y después de 6 meses (T2) de realizada la expansión rápida maxilar.

\begin{tabular}{|c|c|c|c|c|c|c|c|c|}
\hline \multirow{3}{*}{ VARIABLE } & \multicolumn{3}{|c|}{ T1 } & \multicolumn{3}{|c|}{ T2 } & \multicolumn{2}{|c|}{ T1 Vs T2 } \\
\hline & Izq & Der & & Izq & Der & & Izq & Der \\
\hline & Media \pm D.E & Media \pm D.E & $\mathbf{p}$ & Media \pm D.E & Media \pm D.E & $\mathbf{p}$ & $\mathbf{p}$ & $\mathbf{p}$ \\
\hline Longitud Anterior & $1,63 \pm 0,12$ & $1,94 \pm 0,14$ & $0,02^{*}$ & $2,04 \pm 0,14$ & $2,23 \pm 0,20$ & 0,22 & $0,009^{\star}$ & $0,028^{*}$ \\
\hline Longitud Superior & $2,53 \pm 0,20$ & $2,69 \pm 0,26$ & 0,44 & $2,20 \pm 0,15$ & $2,60 \pm 0,23$ & 0,20 & 0,111 & 0,596 \\
\hline Longitud Posterior & $2,37 \pm 0,27$ & $2,36 \pm 0,19$ & $0,76^{\dagger}$ & $1,39 \pm 0,11$ & $1,75 \pm 0,13$ & $0,01^{*}$ & $0,038^{\dagger *}$ & $0,003^{*}$ \\
\hline Posición Condilar & $16,73 \pm 6,64$ & $9,48 \pm 6,42$ & 0,19 & $-18,56 \pm 5,07$ & $-11,37 \pm 6,11$ & 0,14 & $0,000^{*}$ & $0,000^{*}$ \\
\hline
\end{tabular}

t: Prueba de wilcoxon.

$\star: p<0,05$

Fuente: elaboración propia

Tabla 3. Distancia de los espacios condilares en milímetros ( $\mathrm{mm})$, diferenciando el lado donde se presenta de acuerdo con el lado donde se presenta la mordida cruzada posterior unilateral antes (T1) y después de 6 meses (T2) de realizada la expansión rápida maxilar.

\begin{tabular}{ccccccccc}
\hline \multirow{2}{*}{ VARIABLE } & \multicolumn{2}{c}{ T1 } & & & \multicolumn{2}{c}{ T2 } & \multicolumn{2}{c}{ T1 Vs T2 } \\
\cline { 2 - 9 } & Cruzado & No Cruzado & & Cruzado & No Cruzado & & Cruzado & No Cruzado \\
\cline { 2 - 9 } & Media $\pm \mathbf{D . E}$ & Media $\pm \mathbf{D . E}$ & $\mathbf{p}$ & Media $\pm \mathbf{D . E}$ & Media $\pm \mathbf{D . E}$ & $\mathbf{p}$ & $\mathbf{p}$ & $\mathbf{p}$ \\
\hline Longitud Anterior & $1,91 \pm 0,15$ & $1,65 \pm 0,11$ & 0,08 & $2,22 \pm 0,20$ & $2,05 \pm 0,14$ & 0,29 & $0,025^{\star}$ & $0,011^{*}$ \\
\hline Longitud Superior & $2,69 \pm 0,21$ & $2,53 \pm 0,25$ & 0,44 & $2,64 \pm 0,20$ & $2,15 \pm 0,17$ & 0,09 & 0,808 & $0,044^{*}$ \\
\hline Longitud Posterior & $2,24 \pm 0,14$ & $2,49 \pm 0,29$ & 0,26 & $1,69 \pm 0,12$ & $1,46 \pm 0,14$ & 0,15 & $0,002^{*}$ & $0,002^{*}$ \\
\hline Posición Condilar & $8,43 \pm 6,19$ & $17,78 \pm 6,70$ & 0,08 & $-12,72 \pm 5,83$ & $-17,21 \pm 5,57$ & 0,38 & $0,000^{*}$ & $0,000^{*}$ \\
\hline
\end{tabular}

$*: p<0,05$

Fuente: elaboración propia 
Se encontró respecto a la posición del cóndilo en T1 y T2 una concordancia moderada (Kappa= 0 ,5135), De los 9 cóndilos del lado derecho evaluados, 7 se encontraban en una posición anterior y 2 en posición posterior 77,8 \% y 22,2 \% respectivamente); y de los 9 cóndilos del lado izquierdo, 6 se encontraban en una posición anterior, 2 en posición céntrica y 1 en posición posterior $(66,7 \%$, 22,2 \% y 11,1 \% respectivamente) en $T 1$, Y al final del tratamiento (T2) se encontró un aumento y cambio de posición condilar hacia el sector posterior de la cavidad glenoidea tanto en los cóndilos del lado derecho: 3 en posición anterior, 1 en posición céntrica y 5 en posición posterior (33,3\%, 11,1 \% y 55,6 \% respectivamente), como izquierdo ubicándose todos en la zona posterior (100\%).

Tabla 4. Concordancia de la posición condilar entre lado derecho e izquierdo en $\mathrm{T} 1$.

\begin{tabular}{ccc}
\hline Posición condilar & Lado Derecho & Lado Izquierdo \\
\hline Anterior & 7 & 6 \\
\hline Céntrica & 0 & 2 \\
\hline Posterior & 2 & 1 \\
\hline Total & 9 & 9 \\
\hline
\end{tabular}

Kappa $=0,5135$

Fuente: elaboración propia

Tabla 5. Concordancia de la posición condilar entre lado derecho e izquierdo en T2.

\begin{tabular}{ccc}
\hline Posición condilar & Lado Derecho & Lado Izquierdo \\
\hline Anterior & 3 & 0 \\
\hline Céntrica & 1 & 0 \\
\hline Posterior & 5 & 9 \\
\hline Total & 9 & 9
\end{tabular}

Kappa=0.6.0000

Fuente: elaboración propia

Por último, para la expresión del volumen de las vías aéreas en T1, el valor promedio fue de 5,2 cc, y después de realizada la primera fase de tratamiento con ERM, se encontró una leve disminución de la expresión a 5,0cc, pero sin significancia estadística (tabla 6). 
Tabla 6. Expresión del volumen de vías aéreas en T1 y T2 (expresado en cc).

\begin{tabular}{lccc}
\hline \multirow{2}{*}{ Volumen de vías aéreas } & T1 & T2 & \\
\cline { 2 - 4 } & Media \pm D.E & Media \pm D.E & p \\
\cline { 2 - 4 } & $5,211 \pm 2,248$ & $5,000 \pm 1,639$ & 0,5562 \\
\hline
\end{tabular}

Fuente: elaboración propia

\section{Discusión}

En publicaciones anteriores se ha reportado que la autocorrección de la mordida cruzada posterior unilateral varía en un porcentaje de 0 al $10 \%$. De no darse esta autocorrección se producen alteraciones a nivel de las estructuras del sistema estomatognático tales como un desarrollo óseo asimétrico de la rama mandibular del lado afectado, asimetrías mandibulares o faciales, desórdenes a nivel de la ATM, cambios morfológicos y de posición en el cóndilo mandibular, y en la mayoría de los casos se presenta una constricción del arco dental superior donde la expansión rápida maxilar se considera una alternativa terapéutica altamente aceptada $(4,6,10,11,27)$.

Durante el tiempo de tratamiento activo reportado se logró corregir la maloclusión de manera satisfactoria en todos los casos y posteriormente se dejó la aparatología en boca por tres meses más como contención. Este tiempo de retención, según varios autores, va a permitir la reorganización de las suturas maxilares, cumpliendo los objetivos dentales, funcionales y esqueléticos de tratamiento propiciando a la vezun cambio en la posición condilar, así como beneficios de estabilidad oclusal y relajación muscular $(17,18,28,29)$.

La relación entre el cóndilo mandibular y la fosa glenoidea ha sido estudiada por muchos autores (30-32), quienes han demostrado que evaluarla en radiografías convencionales no es tarea fácil, razón por la cual las imágenes obtenidas de tomografías computarizadas tipo Cone Beam se presentan como una herramienta ideal para evaluar la posición condilar en pacientes con mordida cruzada posterior unilateral antes y después de realizado un tratamiento de Ortopedia Maxilar.

En este estudio de pacientes con mordida cruzada posterior unilateral, una vez evaluada la posición condilar antes de la ERM en tomografías computarizadas tipo Cone Beam (TC-CB), se encontró que en el lado afectado existe un aumento del espacio anterior de cóndilo, una disminución leve de la longitud superior y una marcada disminución de la longitud posterior entre el cóndilo y la fosa glenoidea. Esto evidencia un desplazamiento posterior del cóndilo afectado por la maloclusión y, después de realizada la ERM, se encontró un cambio en la posición del cóndilo, algo más posterior, 
y una tendencia leve al cambio de posición en el lado no cruzado, igualmente una posición posterior, razón por la cual se podría mencionar que los cambios obtenidos no afectan de manera negativa el cóndilo (tablas 2 y 3).

Leonardi en el año 2012 (23) realizó un estudio con tomografías computarizadas de baja dosis y analizó los cóndilos antes y después de tratamiento en ambos lados cruzado y no cruzado; y encontró que no se presentaron diferencias estadísticamente significativas en la posición de los cóndilos antes del tratamiento. Pero sí midieron aumentos significativos en el espacio superior de la articulación después del tratamiento en el lado no cruzado, y aumentos relativos en el espacio articular anterior y posterior de este mismo lado (no cruzado). Mientras que el espacio articular posterior solamente se incrementó en el lado cruzado $(12,24)$.

Los últimos estudios publicados reportan que las diferencias posicionales del cóndilo mandibular antes de realizar un tratamiento ortopédico para corregir la maloclusión no presentan cambios o diferencias estadísticamente significativas entre el lado cruzado y el no cruzado. Esto, explicado por autores como Hesse y Wang (12,33), se da gracias a la remodelación compensatoria de la fosa y el cóndilo o la variación en el grosor del disco articular de la ATM, así como la capacidad de adaptación que tiene el disco articular a cualquier alteración causada por cambios oclusales que repercuten en el espacio entre el cóndilo y la fosa $(12,32)$. Por último, los cambios de crecimiento adaptativo tienen un gran potencial para que ocurran si las desviaciones mandibulares ocurrieron como resultado de la maloclusión (12,34).

Es reconocido también por muchos autores que la ERM se asocia con un incremento del volumen total de la vía aérea y del volumen de varias regiones de la vía aérea superior. Sin embargo, en algunas ocasiones se puede registrar una mínima disminución durante el periodo de retención, algo similar a lo registrado en este estudio, pues dichos resultados algo contradictorios y que no son estadísticamente significativos probablemente se deben al poco tiempo trascurrido entre las dos tomas de imágenes, lo cual no permitió que se acentúen los cambios estructurales en los órganos relacionados (35-40).

Por último, es importante recordar que la ERM tiene efectos positivos en las estructuras dentofaciales a nivel de los tres planos del espacio: transversal, sagital y vertical (41), y estos efectos tienen una fuerte asociación con la biometría y las características cefalométricas de los individuos (11), algo a tener en cuenta para entender no solo los resultados a corto o largo plazo sino la estabilidad de los mismos en la transición de la dentición mixta a la dentición permanente. Así como el posicionamiento durante la toma de la TC-CB, ya que si no lo realiza un único operador, tal como lo realizado en este estudio, se podría afectar la posición condilar, razón por la cual 
se recomienda una estandarización de la posición tomográfica siguiendo el método propuesto por los autores Leonardi e Ikeda $(23,24)$.

\section{Conclusiones}

Realizar un tratamiento de ortopedia maxilar en edades tempranas ayuda a interceptar o prevenir las asimetrías morfológicas favorecidas por el desplazamiento mandibular que presentan los pacientes con mordida cruzada posterior unilateral.

La intervención oportuna para corregir las anomalías transversales en el paciente pediátrico favorece no solo el desarrollo armónico de los maxilares sino de todo el complejo craneofacial en general. El protocolo aquí descrito proporcionó cambios a nivel funcional, dental y esquelético, coincidentes con lo reportado previamente en la literatura, y a la vez generó un impacto positivo y significativo en la autoestima de los pacientes tratados, mejorando la estética de la sonrisa, incrementando la dimensión transversal del arco dental superior que permite corregir las discrepancias de tamaño entre las bases óseas y los dientes.

- De no ser tratada a tiempo, la mordida cruzada posterior unilateral puede generar en máxima intercuspidación una posición condilar asimétrica en el lado afectado, acompañado de un desplazamiento y aumento en el espacio articular del lado no afectado. Gracias a TC-CB se pudo obtener una imagen clara y precisa donde se evaluó la posición condilar en condiciones tanto de maloclusión (lado afectado) como de normoclusión (lado no afectado). Esta información es de gran ayuda desde el punto de vista morfológico y funcional, ya que permite evaluar posición ideal del cóndilo mandibular, y junto con el tratamiento de ortopedia maxilar se evita que la rama mandibular genere adaptaciones en una función anormal, así como posiciones posturales que generalmente son asimétricas.

\section{Recomendaciones}

Los resultados de este articulo corresponden a la primera fase de la investigación (seis meses), se está desarrollando una segunda fase equivalente a un año después de realizado el tratamiento. Por esta razón se recomienda un tiempo de seguimiento adicional para evaluar estabilidad de cambios generados en la posición condilar y vías aéreas en estudios futuros. 
Se requiere una investigación con tamaño de muestra más amplio y con rango de edad mayor para evaluar los cambios estructurales que sufre el cóndilo mandibular en pacientes que presenten una mordida cruzada posterior unilateral.

\section{Conflicto de intereses}

Los autores afirman que no tienen conflicto de intereses.

\section{Referencias}

1. Colombia. Ministerio de Salud. IV Estudio Nacional de Salud Bucal (ENSAB IV). Situación en Salud Bucal. Tomo I. Bogotá: Ministerio de Salud; 2014.

2. Espinal-Botero G, Muñoz A, Flores L, Ponce M, Nava J, González J. Frecuencia de maloclusión en las clínicas odontopediátricas de la Universidad de Antioquia, Colombia, y de la Universidad Autónoma de San Luis Potosí, México. Rev Nac Odontol. 2016;12(22):61-68. doi: http://dx.doi.org/10.16925/od.v12i22.1206

3. Moyers R. Handbook of orthodontics. 2nd ed. Chicago: Yearbook Medical Publishers; 1966.

4. Mora N, Hernández JA, Rodríguez C. Alternative of timely treatment of unilateral posterior cross-bite in primary and mixed early dentition. Case series. Revista Estomatología. 2019;27(1):43-55. doi: https://doi.org/10.25100/re.v27i1.8407

5. Iodice G, Danzi G, Cimino R, Paduano S, Michelotti A. Association between posterior crossbite, skeletal, and muscle asymmetry: a systematic review. Eur J Orthod. 2016;38(6):638-651. doi: https://doi.org/10.1093/ejo/cjw003

6. Kennedy DB, Osepchook M. Unilateral Posterior Crossbite With Mandibular Shift: A Review. J Can Dent Assoc. 2005; 71:569-573.

7. Marshall SD, Southard KA, Southard TE. Early transverse treatment. Semin Orthod. 2005;11(3):130-9. doi: https://doi.org/10.1053/j.sodo.2005.04.006

8. UNAL. Guía de atención de mordidas cruzadas. Bogotá: Universidad Nacional de Colombia; 2016. 
Evaluación de la posición condilar y de las vías aéreas en niños con mordida cruzada posterior unilateral antes y después de tratamiento con férula de expansión. Parte 1

9. Bell RA, Kiebach TJ. Posterior crossbites in children: Developmental-based diagnosis and implications to normative growth patterns. Semin Orthod. 2014;20(2):77-113. doi: https://doi. org/10.1053/j.sodo.2014.04.002

10. Pinto AS, Buschang PH, Throckmorton GS, Chen P. Morphological and positional asymmetries of young children with functional unilateral posterior crossbite. Am J Orthod Dentofacia IOrthop. 2001;120(5):513-20. doi: https://doi.org/10.1067/mod.2001.118627a

11. ThilanderB, Lennartsson B.AStudy of Children With Unilateral PosteriorCrossbite, Treated and Untreated, in the Deciduous Dentition--Occlusal and Skeletal Characteristics of Significance in Predicting the Long-Term Outcome. J Orofac Orthop. 2002;63(5):371-83. doi: https://doi. org/10.1007/s00056-002-0210-6

12. Pittman L, Shipley TS, Martin CA, Xiang J, Ngan PW. CBCT evaluation of condylar changes in children with unilateral posterior crossbites and a functional shift. Semin Orthod. 2019;25(1):36-45. doi: https://doi.org/10.1053/j.sodo.2019.02.005

13. Ortu E, Pietropaoli D, Ortu M, Giannoni M, Monaco A. Evaluation of cervical posture following rapid maxillary expansion: a review of the literature. Open Dent J. 2014;8:20-7. doi: https://doi. org/10.2174/1874210601408010020

14. Bjerklin K. Follow-up control of patients with unilateral posterior cross-bite treated with expansion plates or the quad-helix appliance. J Orofac Orthop. 2000;61(2):112-124. doi: https:// doi.org/10.1007/bf01300353

15. Bindayel NA. Simple Removable Appliances to Correct Anterior and Posterior Crossbite in Mixed Dentition: Case Report. Saudi Dent J. 2012;24(2):105-13. doi: https://doi.org/10.1016/j. sdentj.2011.12.005

16. Valencia RM. Treatment of Unilateral Buccal Crossbites in the Primary, Early Mixed, and Permanent Dentitions: Case Reports. J Pediatr Dent. 2007;31(3): 214-218.

17. Ramoglu SI, Sari Z. Maxillary expansion in the mixed dentition Rapid or semi-rapid? Eur J Orthod. 2010; 32:11-18. doi: https://doi.org/10.1093/ejo/cjp057

18. McNamara JJA, Franchi L, McClatchey LM. Orthodontic and orthopedic expansion of the transverse dimension: A four-decade perspective. Semin Orthod. 2019;25(1):3-15. doi: https:// doi.org/10.1053/j.sodo.2019.02.002 
19. Furlan FL, Beti MM. Un tratamiento antiguo y vigente en ortodoncia. Expansión Rápida Maxilar. Rev Soc Odontol. 2015; 25(51):5-14.

20. Rodríguez de Guzmán J, Talens Cogollos L, Álvarez Vila N, Tarazona Álvarez B, Zamora Martínez N, Gandía Franco Jl. Estudio de los cambios en la posición natural de la cabeza tras la expansión rápida del maxilar. Rev Esp Ortod. 2015; 45(3):136-41.

21. Liu S, Xu T, Zou W. Effects of rapid maxillary expansion on the midpalatal suture: a systematic review. Eur J Orthod. 2015;37(6):651-655.

22. Ikeda K, Kawamura A. Assessment of optimal condylar position with limited cone-beam computed tomography. Am J Orthod Dentofacial Orthop. 2009;135(4):495-501. doi: https:// doi.org/10.1016/j.ajodo.2007.05.021

23. Leonardi R, Caltabiano M, Cavallini C, Sicurezza E, Barbato E, Spampinato C, et al. Condyle fossa relationship associated with functional posterior crossbite, before and after rapid maxillary expansion. Angle Orthod. 2012;82(6):1040-1046. doi: https://doi.org/10.2319/112211-725.1

24. Hesse KL, Artun J, Joondeph DR, Kennedy DB. Changes in Condylar Position and Occlusion Associated With Maxillary Expansion for Correction of Functional posterior Crossbite. Am J Orthod Dentofacial Orthop. 1997;111(4):410-8. doi: https://doi.org/10.1016/ s0889-5406(97)80023-6

25. Pullinger AG, Hollender L, Solberg WK, Petersson A. A Tomographic Study of Mandibular Condyle Position in an Asymptomatic Population. J Prosthet Dent. 1985;53(5):706-13. doi: https://doi.org/10.1016/0022-3913(85)90029-0

26. Chang Y, Koenig LJ, Pruszynski JE, Bradley TG, Bosio JA, Liu D. Dimensional Changes of Upper Airway After Rapid Maxillary Expansion: A Prospective Cone-Beam Computed Tomography Study. Am J Orthod Dentofacial Orthop. 2013;143(4):462-70. doi: https://doi.org/10.1016/j. ajodo.2012.11.019

27. ] Marco Cambra V, Jiménez Arcos G, Gatón Hernández P, De Castañeda Regojo ER. Las cuatro claves del tratamiento orofacial en crecimiento. Rev Esp Ortod. 2020;50(1):10-24.

28. Cozzani M, Mazzotta L, Cozzani P. Early interceptive treatment in the primary dentition - a case report. J Orthod. 2013;40(4):345-51. doi: https://doi.org/10.1179/1465313313y.0000000068

29. McNamara J, Seligman D, Okeson J. Occlusion, orthodontic treatment and temporomandibular disorders: a review. J Orofac Pain. 1995;9(1):73-90. 
Evaluación de la posición condilar y de las vías aéreas en niños con mordida cruzada posterior unilateral antes y después de tratamiento con férula de expansión. Parte 1

30. Tsuruta A, Yamada K, Hanada K, Hosogai A, Kohno S, Koyama J, Hayashi T. The relationship between morphological changes of the condyle and condylar position in the glenoid fossa. J Orofac Pain. 2004;18(2):148-55.

31. Herrera-Guardiola S, Puerta-Salazar GE, Martínez Cajas CH. Condylar position changes after three months of treatment with posterior bite turbo. Rev Fac Odontol Univ Antioq. 2020;32(1):18-25. doi: http://dx.doi.org/10.17533/udea.rfo.v32n1a2

32. Hesse KL, Artun J, Joondeph DR, Kennedy DB. Change in condylar position and occlusion associated with maxillary expansion for correction of functional unilateral posterior crossbite. Am J Orthod Dentofacial Orthop. 1997;111(4):410-418. doi: https://doi.org/10.1016/ s0889-5406(97)80023-6

33. Wang MQ, He JJ, Li G, Widmalm SE. The effect of physiological non balanced occlusion on the thickness of the temporomandibular joint disc: a pilot autopsy study. J Prosthet Dent. 2008;99(2):148-52. doi: https://doi.org/10.1016/s0022-3913(08)60031-1

34. Myers DR, Barenie JT, Bell RA, Williamson EH. Condylar position in children with functional posterior crossbites: before and after crossbite correction. Pediatr Dent. 1980;2(3):190-4.

35. Pérez AP.El papel del ortodoncista en la apnea obstructiva del sueño en el niño. Rev Esp Ortod. 2018;48(1):3-4.

36. Buck LI M, Dalci O, Darendeliler MA, Papageorgiou SN, Papadopopulou AK. Volumetric upper airway changes after rapid maxillary expansion: a systematic review and meta-analysis. Eur J Orthod. 2017;39(5):463-73.

37. Niu X, Di Carlo G, Cornelis MA, Cattaneo PM. Three-dimensional analyses of short- and long-term effects of rapid maxillary expansion on nasal cavity and upper airway: A systematic review and meta-analysis. Orthod Craniofac Res. 2020;23(3):250-276. doi: https://doi. org/10.1111/ocr.12378

38. White SM, Huang CJ, Huang SC, Sun Z, Eldredge JD, Mallya SM. Evaluation of the upper airway morphology: the role of cone beam computed tomography. J Calif Dent Assoc. 2015;43(9):531-539.

39. Di Carlo G, Saccucci M, lerardo G, Luzzi V, Occasi F, Zicari AM, et al. Rapid Maxillary Expansion and Upper Airway Morphology: A Systematic Review on the Role of Cone Beam Computed Tomography. Biomed Res Int. 2017; 2017:5460429. doi: https://doi.org/10.1155/2017/5460429 
40. Bucci R, Montanaro D, Rongo R, Valletta R, Michelotti A, D’Anto V. Effects of maxillary expansion on the upper airways: Evidence from systematic reviews and meta-analyses. J Oral Rehabil. 2019;46(4):377-387. doi: https://doi.org/10.1111/joor.12766

41. Ramoglu SI, Sari Z. Maxillary expansion in the mixed dentition: rapid or semi-rapid? Eur J Orthod. 2010;32(1):11-8. doi: https://doi.org/10.1093/ejo/cjp057 